\title{
EchoGéo
}

38 | 2016

Varia

Potentialités de l'outil LiDAR pour cartographier les vestiges de la Grande Guerre en milieu intraforestier (bois des Caures, forêt domaniale de Verdun, Meuse)

Rémi de Matos Machado, Jean-Paul Amat, Gilles Arnaud-Fassetta and François Bétard

(2) OpenEdition

Journals

Electronic version

URL: https://journals.openedition.org/echogeo/14791

DOI: 10.4000/echogeo.14791

ISSN: 1963-1197

Publisher

Pôle de recherche pour l'organisation et la diffusion de l'information géographique (CNRS UMR 8586)

Electronic reference

Rémi de Matos Machado, Jean-Paul Amat, Gilles Arnaud-Fassetta and François Bétard, "Potentialités de l'outil LiDAR pour cartographier les vestiges de la Grande Guerre en milieu intra-forestier (bois des Caures, forêt domaniale de Verdun, Meuse)", EchoGéo [Online], 38 | 2016, Online since 13 December 2016, connection on 31 July 2021. URL: http://journals.openedition.org/echogeo/14791 ; DOI: https:// doi.org/10.4000/echogeo.14791

This text was automatically generated on 31 July 2021.

EchoGéo est mis à disposition selon les termes de la licence Creative Commons Attribution - Pas d'Utilisation Commerciale - Pas de Modification 4.0 International (CC BY-NC-ND) 


\section{Potentialités de l'outil LiDAR pour cartographier les vestiges de la Grande Guerre en milieu intra- forestier (bois des Caures, forêt domaniale de Verdun, Meuse)}

Rémi de Matos Machado, Jean-Paul Amat, Gilles Arnaud-Fassetta and François Bétard

Les auteurs tiennent à remercier Stéphanie Jacquemot, de la DRAC ACAL (Alsace-ChampagneArdennes-Lorraine) - Service archéologique de Metz, Gersende Gérard, Gérald Colin, René-Marc Pineau et Juliette Foltier, de l'Office national des Forêts - Agence de Verdun, Clélia Bilodeau, de l'université Paris-Diderot (Paris 7), UMR 7533 LADYSS, pour la mise à disposition des connaissances et des moyens techniques et logistiques nécessaires à la réalisation de l'étude, ainsi que Denis Jacquemot, Romain Perrier et Théo Wohlschlegel, pour leur aide apportée sur le terrain. Nous remercions aussi les trois relecteurs pour leurs remarques utiles et constructives qui ont permis d'améliorer le manuscrit.

\section{Introduction}

1 Les quatre années (2014-2018) de commémorations nationales et internationales du centenaire de la Première Guerre mondiale donnent aux recherches sur les paysages de guerre une importance toute particulière. Faisant écho à des enjeux mémoriels et culturels d'actualité, l'étude des séquelles du premier conflit mondial dans les paysages $\mathrm{du}$ Nord et du Nord-Est de la France répond à une forte demande sociale et politique en termes d'enjeux environnementaux et patrimoniaux (Desfossés et al., 2008 ; Jacquemot et Legendre, 2011; Gheyle et al., 2013; Schnitzler et Landolt, 2013 ; Stichelbaut et Chielens, 2013 ; Amat, 2015). C'est en particulier le cas en Meuse où l'obtention récente du label «Forêt d'Exception ${ }^{\circledast}$ " pour la forêt domaniale de Verdun a engagé les acteurs 
locaux (élus, gestionnaires, associations) dans une démarche d'excellence autour de la gestion du patrimoine forestier, culturel et paysager, dans une perspective de développement territorial durable (Amat, 2015). Une meilleure connaissance du champ de bataille sous forêt établit le socle scientifique d'une gestion conservatoire des milieux, préalable à toute démarche de valorisation du patrimoine archéologique et environnemental de ce site historique (Stichelbaut, 2009, 2011 ; Doneus et Briese, 2011 ; Georges-Leroy et al., 2011 ; Stichelbaut et Chielens, 2013). Les traces géomorphologiques et archéologiques (trous d'obus, tranchées, abris, etc.) masquées par le couvert végétal sont en effet menacées d'effacement (érosion, fréquentation touristique, fouilles clandestines, gestion sylvicole), d'où l'importance de les inventorier et de les cartographier précisément. Une mission LiDAR (Light Detection and Ranging) aéroportée a permis de prospecter en 2013 l'ensemble des 10000 hectares de la forêt domaniale de Verdun, dévoilant sous forme d'images 3-D à haute résolution la plupart des vestiges de guerre dissimulés sous le couvert forestier.

2 Cet article propose d'évaluer les potentialités de ces données de télédétection pour remettre au jour les vestiges de la Grande Guerre présents sur les 70 hectares du bois des Caures, célèbre pour la résistance héroïque des bataillons de chasseurs à pied les 21 et 22 février 1916 (Stéphane, 1929; Bernède, 2002). Une méthodologie innovante permet un inventaire et une cartographie détaillée des infrastructures du front enterré dans ce secteur. Elle se fonde sur l'utilisation combinée de la télédétection, des observations de terrain et des archives historiques. En effet, les combats du bois des Caures ont laissé dans le paysage des traces nettement marquées, en créant des formes de relief anthropogéniques encore peu étudiées en tant que telles (Arnould et Simon, 1994 ; Amat, 2001 ; Hupy, 2005 ; Hupy et Schaetzl, 2006 ; Ilyès, 2006 ; Desfossés et al., 2008 ; Jacquemot et Legendre, 2011 ; Schnitzler et Landolt, 2013 ; Hesse, 2014 ; MassonLoodts, 2014). Replacé dans le contexte plus général du "Front de l'Ouest» (illustration 1), ce site d'étude offre un exemple démonstratif de l'impact encore visible de l'action des soldats sur les milieux physiques, un siècle après la bataille de Verdun. 


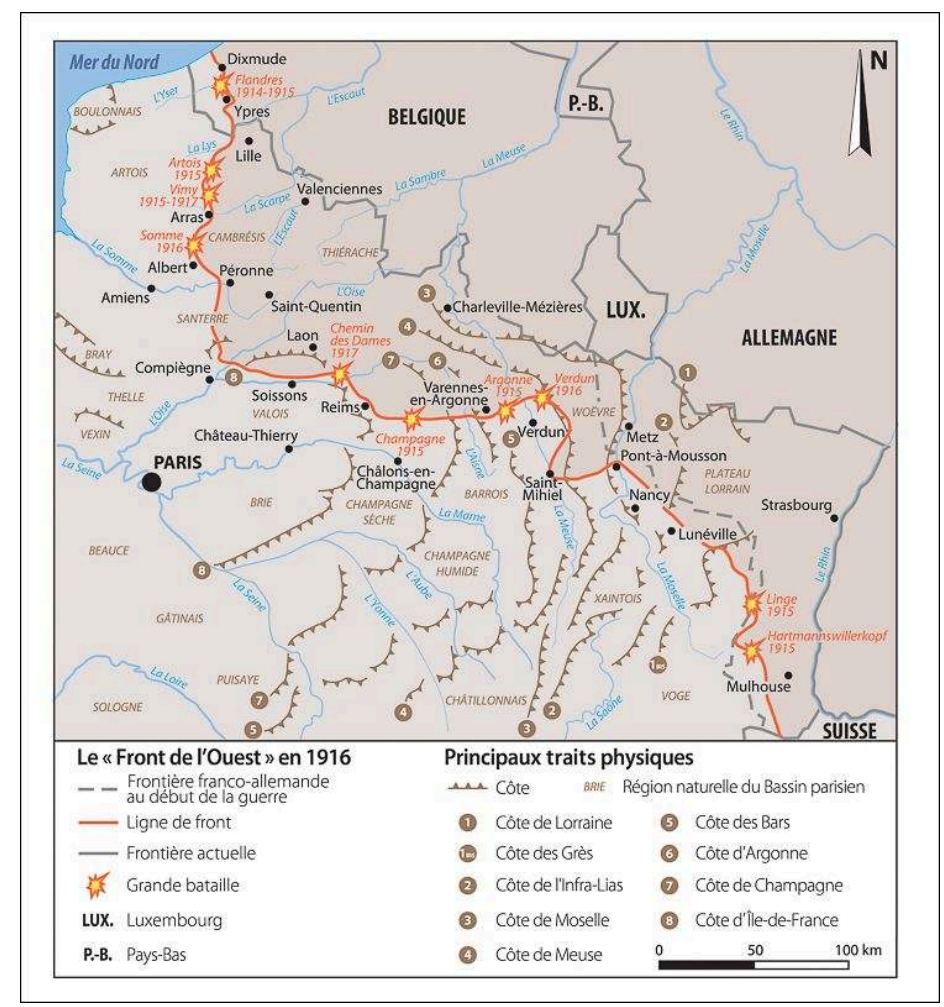

L'hiver 1914 marqua le passage à la « guerre de position ». La ligne de front résultant de cette stabilisation varia peu jusqu'aux derniers mois du conflit.

Bien que l'étude des dimensions environnementales de la guerre ait suscité ces dernières années un engouement certain de la communauté scientifique, peu de travaux se sont intéressés aux "polémoformes", terme employé pour désigner les formes de relief et les modelés nés de la guerre (Amat, 1987, 2001). On peut citer :

- Les travaux de Jean-Paul Amat sur les forêts de guerre de l'arc meusien (front de l'Argonne à la Woëvre) présentent des réflexions sur les dynamiques pédogénétique et géomorphologique du plateau de Douaumont (forêt domaniale de Verdun, illustration 2), nom donné aux 193 hectares de l'espace domanial maintenus hors aménagement forestier (Amat et de Foucault, 1999 ; Amat, 2001, 2015) ;

- Les recherches de l'université de Reims sur la cartographie des réseaux de défense de la région rémoise et les relations entre le milieu physique et l'organisation du champ de bataille (Devos et al., 2015) ;

- La thèse de Joseph Hupy a contribué à l'émergence du concept de «bombturbation » et propose une approche morphométrique des trous d'obus en forêt domaniale de Verdun (Hupy, 2005 ; Hupy et Schaetzl, 2006) ;

- Les recherches de David Passmore portent sur la caractérisation morphométrique et archéologique des vestiges de guerre du deuxième conflit mondial dans la forêt normande des Andaines (Passmore et al., 2013a, 2013b) ;

- Enfin, les travaux de l'université de Gand ont enrichi la connaissance du front flamand de 1914-1918, en exploitant les photographies aériennes de l'époque pour cartographier et quantifier les installations militaires par le biais des SIG (Stichelbaut, 2009, 2011; Gheyle et al., 2013 ; Stichelbaut et Chielens, 2013 ; Van Hollebeeke et al., 2014). 
Inscrite dans la continuité de ces travaux, cette étude propose une nouvelle approche d'inventaire à partir de l'imagerie LiDAR. De plus, la recherche s'inscrit dans une démarche géoarchéologique, qui fait appel à la géomorphologie (description morphologique), à l'archéologie (essai d'interprétation) et à l'histoire (combats de 1914-1918, archives historiques - Wandsnider, 1992; Thornbush, 2012; ArnaudFassetta et Carcaud, 2015).

5 Pour comprendre l'origine des polémofaciès du bois des Caures, l'article identifie les dynamiques pendant la Grande Guerre et en période post-conflit. L'analyse présente la méthode d'inventaire et de cartographie des vestiges de guerre dans le bois. L'imagerie LiDAR décrit puis interprète le champ de bataille à l'aide des observations de terrain et des documents d'archives (plans historiques, photographies aériennes), afin d'apprécier le degré de préservation de ces vestiges compte tenu de la chronologie de leur genèse pendant les années de guerre. L'analyse s'achève par une discussion des potentialités de la technologie LiDAR pour conserver et valoriser le patrimoine historique de la forêt domaniale de Verdun.

\section{Le bois des Caures, objet d'étude}

\section{Cadre physique}

La forêt domaniale de Verdun (10 000 hectares environ) se situe à quelque $200 \mathrm{~km}$ à l'est de Paris (département de la Meuse, région Grand Est) et borde la ville de Verdun au nord-est. Elle s'inscrit dans le relief de cuestas de l'Est du Bassin parisien au niveau des Côtes de Meuse (illustrations 1 et 2). Elle occupe le plateau des Hauts de Meuse, compris en moyenne entre 300 et $350 \mathrm{~m}$ (illustration 3). La forêt est bordée 1) à l'ouest, par la vallée de la Meuse, encaissée d'environ 150-200 m et 2) à l'est et au nord, par la plaine de la Woëvre, aux altitudes moyennes comprises entre 200 et $250 \mathrm{~m}$. Le commandement de la Côte de Meuse, talus convexo-concave fortement échancré, est de 80 à $110 \mathrm{~m}$. Le substrat présente une alternance de couches résistantes (bancs de calcaire massif) et tendres (argiles et marnes), datés du Callovien à l'Oxfordien supérieur. La base de la cuesta des Côtes de Meuse est formée par une épaisse couche de marnes et d'argiles silteuses du Callovien. Le front des Côtes de Meuse se dégage dans un binôme de calcaires et de marnes de l'Oxfordien inférieur et moyen dans lesquelles s'interposent à la limite des deux sous-étages des argiles à chailles et des oolithes ferrugineuses. Sur le plateau affleure les calcaires et marnes de l'Oxfordien moyen puis un second ensemble composé de calcaires massifs de l'oxfordien supérieur. Le plateau est coiffé par des placages argileux recouverts de calcaires et marnes de l'Oxfordien supérieur (figures 3a et 3b - Enay et Boullier, 1981 ; Amat, 2001 ; Carpentier, 2004). 
Illustration 2 - Situation du bois des Caures dans la forêt domaniale de Verdun

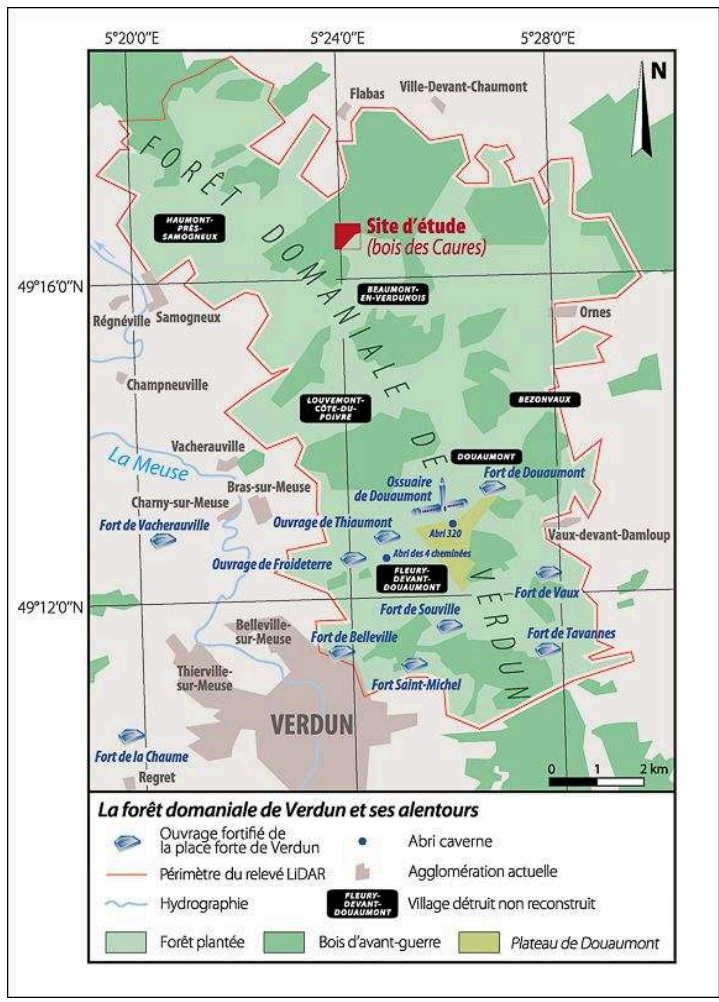

Illustration 3 - Carte géomorphologique de la forêt domaniale de Verdun au 1:80.000

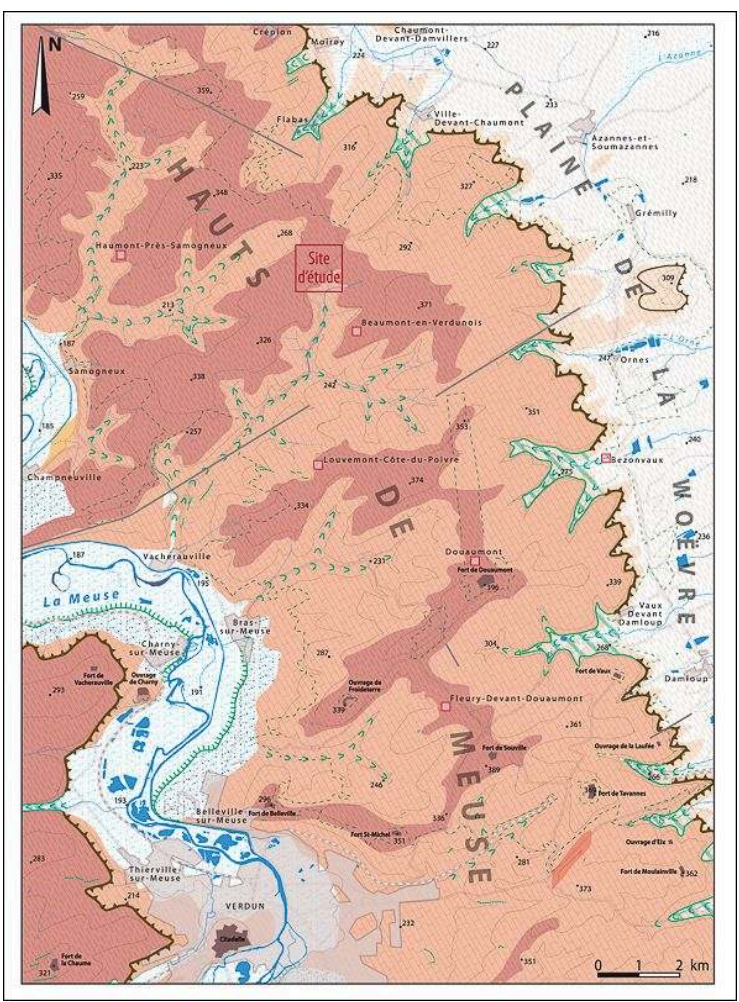




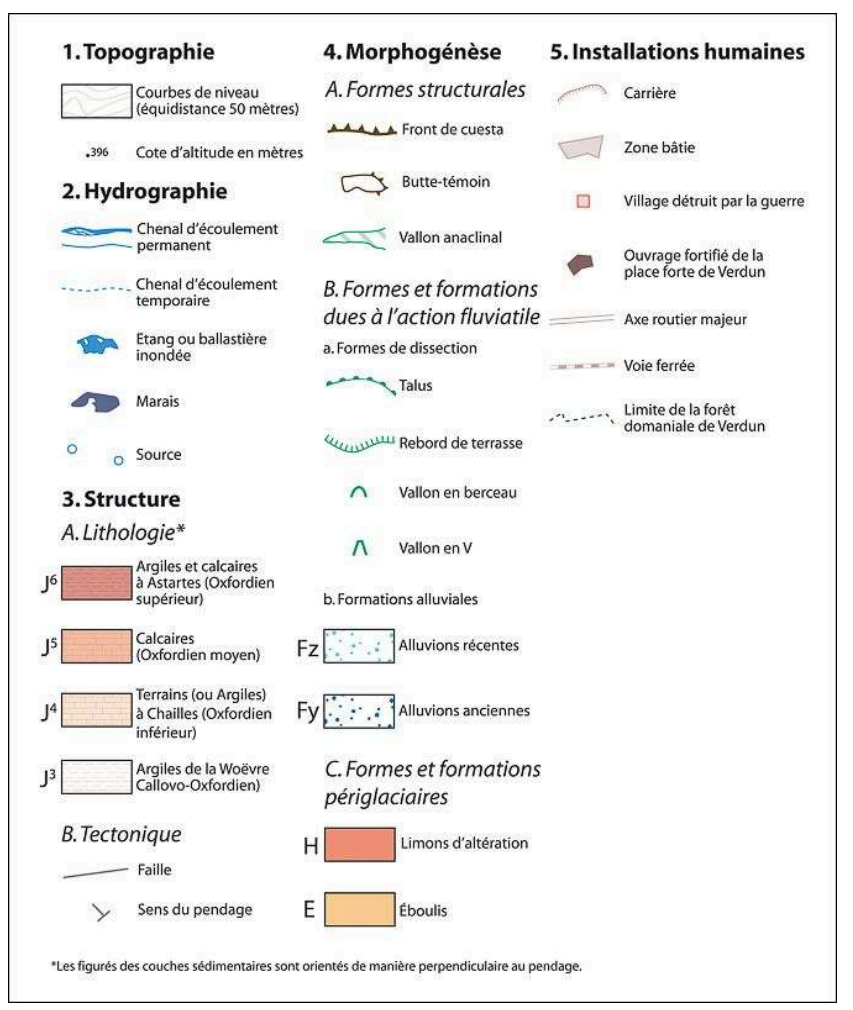

La carte a été dessinée à partir de la carte topographique : Forêts de Verdun et du Mort Homme (TOP 25 - 3112 ET). Saint-Mandé : IGN, 2014 ; et des cartes géologiques de la France à 1/50 000 : Verdunsur-Meuse (feuille 135). Orléans : BRGM, 1969 et Étain (feuille 136). Orléans : BRGM, 1958. Les données LiDAR ont servi au traçage des courbes de niveau et à l'identification de la morphologie des vallées.

7 Le bois des Caures occupe la limite septentrionale de la forêt de Verdun (illustration 2). En 1914, il porte des peuplements de taillis sous futaie feuillus, en grande partie détruits au début de la bataille de Verdun (1916). Une restauration spontanée marque l'immédiat d'après-guerre (Amat, 2001). Son assise court sur une ligne de crête orientée sud-ouest/nord-est culminant à $340 \mathrm{~m}$ et limitée par deux ravins : 1) au nord-ouest, le ravin du bois des Caures (entre 220 et $270 \mathrm{~m}$ ) et 2) au sud-est, le ravin du Fays (entre 250 et $280 \mathrm{~m}$ ). De larges placages argileux et marno-calcaires de l'Oxfordien supérieur entretiennent une nappe phréatique perchée, qui donne naissance à des sources de débordement. La violence caractéristique de la guerre de position et la répétition des bombardements d'artillerie sur un espace frontal large de quelques kilomètres ont engendré des faciès inédits (illustration 4). Comme ailleurs dans la forêt de Verdun, le bois des Caures présente une micro-topographie bosselée et des empreintes nombreuses et variées : trous d'obus, tranchées, abris, dépôts de munitions ou encore plates-formes de voies ferrées (Doyle et Bennett, 1997 ; Bernède, 2002). 
Illustration 4 - Vue vers le sud du champ de bataille de Verdun depuis l'abri 320

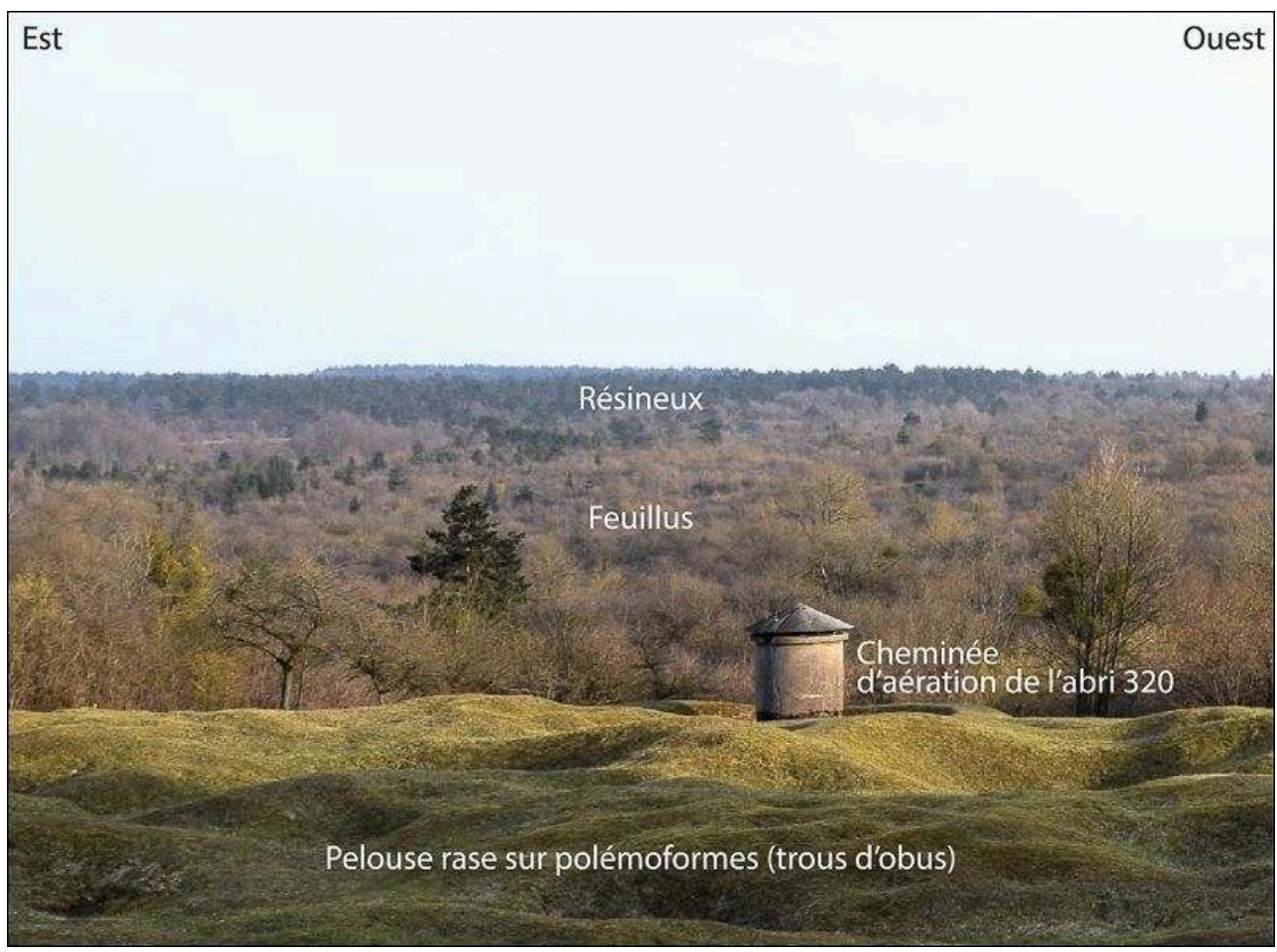

La photographie représente un des seuls points de vue de la forêt permettant d'apprécier à la fois la topographie bouleversée par les combats et les différents peuplements forestiers de la forêt domaniale de Verdun. II s'agit de l'abri caverne 320, situé à quelques encablures (au sud) de l'ossuaire de Douaumont. Au premier plan apparait un relief criblé d'impacts d'obus qui se sont abattus sur l'abri caverne 320. Au second plan, le Plateau de Douaumont (zone en libre évolution depuis la fin de la guerre) et à l'arrière-plan, les peuplements gérés, feuillus et résineux.

Auteur : R. de Matos Machado, mars 2015.

\section{Cadre historique}

8 La forêt domaniale de Verdun est un haut lieu de mémoire du Front de l'Ouest. Sur ce théâtre d'un des plus grands affrontements de l'Histoire, la bataille dura dix mois (du 21 février au 18 décembre 1916). Le bois des Caures fut le seul secteur ayant résisté aux deux millions d'obus tirés et aux milliers de fantassins allemands lancés le 21 février 1916 sur un front large de $5 \mathrm{~km}$ et profond de $2 \mathrm{~km}$ (Amat, 2001; Bernède, 2002). Au cœur de cette défense héroïque, les $56^{\mathrm{e}}$ et $59^{\mathrm{e}}$ bataillons de chasseurs à pied du lieutenant-colonel Driant, arc-boutés aux restes de tranchées de la première position (Stéphane, 1929; Bernède, 2002). Au deuxième jour de l'offensive, les Allemands s'emparent du bois mais n'en réorganisent pas les défenses, les combats se déplaçant au sud. À la fin de la bataille, le 18 décembre, le bois des Caures se retrouve sur la première position allemande d'un front qui se stabilise à nouveau. Le bilan de ces « 300 jours » d'affrontement est lourd: plus de 700000 victimes dont 300000 tués et disparus (Bernède, 2002). En 1917, les Allemands aménagent solidement le bois. Les travaux se poursuivent en 1918 jusqu'à l'offensive franco-américaine «MeuseArgonne ».

Dans l'immédiat après-guerre, l'État français planifie la remise en état des terres dévastées. Après nettoiement du champ de bataille, les secteurs définitivement expropriés de la zone rouge, terres agricoles, bois et forêts, sont dévolus au ministère 
de l'Agriculture aux fins de boisement (Clout, 1993 ; Amat, 2001, 2015 ; Arnould, 2006). Entre 1929 et 1935, plus de 36 millions d'arbres sont plantés, sans nivellement, dans l'objectif de créer une forêt de résineux. Les bois d'avant-guerre, tel le bois des Caures, se reconstituent sans intervention de l'Homme. Un siècle plus tard, un vaste manteau forestier (la forêt domaniale de Verdun) recouvre le champ de bataille composé d'un tiers de résineux et de deux tiers de feuillus (illustrations 2 et 4 - Amat, 1987, 2001, 2015). L'émergence de ce massif de près de 10000 hectares a largement contribué à conserver les vestiges de guerre, par l'action protectrice du couvert sur les sols (interception et absorption des gouttes de pluie par la canopée, la litière et le réseau racinaire - Rey, 2003; Meuret, 2010). La forêt domaniale de Verdun a conservé la mémoire de l'un des plus vastes champs de bataille de la Grande Guerre; tout cheminement permet d'observer les multiples traces des combats (Jacquemot et Legendre, 2011 ; Schnitzler et Landolt, 2013).

Parallèlement aux commémorations du centenaire, la forêt domaniale de Verdun s'est vue attribuer le 20 juin 2014 le label «Forêt d'Exception ${ }^{\circledast}$ ». Créé en 2007 par l'ONF (Office national des Forêts), le label vise à distinguer les forêts domaniales françaises les plus remarquables du point de vue de leur patrimoine naturel et culturel, dans une démarche d'exemplarité en matière de développement durable. C'est dans ce cadre qu'une mission de télédétection par laser aéroporté a été cofinancée par l'ONF (agence de Verdun), le Service Régional de l'Archéologie de Lorraine, le Département de la Meuse et le GIP «Objectif Meuse », afin d'établir une cartographie précise des vestiges de guerre dissimulés par la forêt (Amat, 2015).

\section{Données et méthodes}

\section{L'imagerie LiDAR : principe d'acquisition et traitement des données}

11 La première étape de la démarche méthodologique a eu pour objectif l'exploitation d'images LiDAR. Dans le domaine de l'archéologie, cette technologie a été employée pour la première fois en France dans la forêt de Haye pendant l'hiver 2006-2007 (Georges-Leroy et al., 2011). Depuis, elle n'a cessé d'être utilisée par les archéologues, notamment pour sa capacité à traverser le manteau forestier et à faire abstraction du bruit atmosphérique (Bilodeau et al., 2008; Doneus et Briese, 2011 ; Georges-Leroy et al., 2011 ; Masini et al., 2011). L'acquisition des données LiDAR en forêt domaniale de Verdun a été réalisée par l'entreprise Sintégra, les 26 et 27 mars 2013 par envoi vers le sol d'impulsions laser depuis un avion situé à environ $600 \mathrm{~m}$ d'altitude. Les temps de retour des échos résultant de l'interception du signal par les différentes catégories d'objets présents au sol, permettent d'estimer l'altitude du point d'impact et de mesurer la position des objets interceptés (Bilodeau et al., 2008 ; Georges-Leroy et al., 2011 ; Robert, 2011). Sur près de $115 \mathrm{~km}^{2}$ (illustration 2) plus de 5 milliards de mesures altimétriques ont été enregistrées, à raison d'une densité moyenne de 16 points enregistrés au sol par mètre carré (44 pts $/ \mathrm{m}^{2}$ avant filtrage). Un travail de reclassification à partir d'algorithmes de filtrage a permis d'obtenir une image continue de la topographie sous la forme d'un modèle numérique de terrain (MNT) d'une résolution de $20 \mathrm{~cm}$, permettant d'apprécier toutes les aspérités du relief.

La cartographie des vestiges de guerre s'est fondée sur le MNT exploité sur les logiciels de traitement d'images et les systèmes d'information géographique (SIG) ArcGIS ${ }^{\circ}$, IDL 
Software ${ }^{\odot}$ et SAGA GIS ${ }^{\odot}$. Toutefois, certains vestiges n'ont pas pu être détectés pour plusieurs raisons : dimension inférieure à la taille des pixels, morphologie biaisée par des objets d'une autre nature, structures enfouies, etc. L'exploitation du MNT a consisté à utiliser différentes techniques de visualisation afin de repérer et cartographier les vestiges et mieux apprécier leur morphologie (illustration 5). Certaines sont particulièrement adaptées à la détection de reliefs très atténués (i.e., parfois $<50 \mathrm{~cm}$ ), notamment l'indice Sky-View Factor qui permet de détecter facilement les formes creuses en calculant les portions de ciel visible pour chaque pixel du MNT (Kokalj et al., 2011 ; Zakšek et al., 2011 ; Štular et al., 2012). La technique du modèle de relief local (Local Relief Model ou residual relief) est tout aussi efficace, à la différence qu'elle prend en considération la position des pixels par rapport aux altitudes avoisinantes (illustration 5 - Hesse, 2010 ; Štular et al., 2012).

Illustration 5 - Synopsis des différentes méthodes de visualisation employées pour la détection des vestiges de guerre

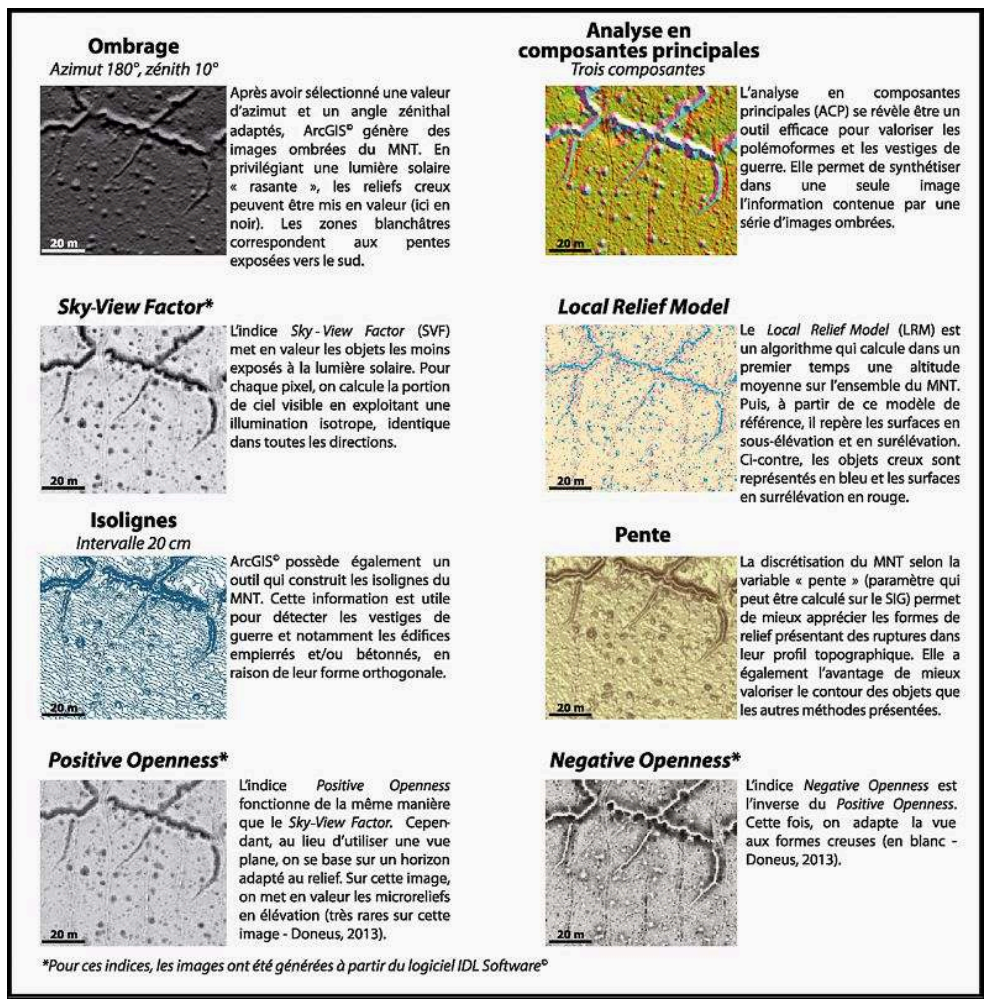

\section{Relevé archéologique et analyse morphologique des vestiges de guerre}

Si les données LiDAR multiplient les découvertes archéologiques en milieu forestier, les observations de terrain et de repérage in situ au DGPS aident à l'identification et à l'interprétation des structures répertoriées (Passmore et al., 2013a). Elles ont été menées dans le bois des Caures en mars et avril 2014, période phénologique où l'absence de feuillage des peuplements caducifoliés offre des conditions de visibilité optimale (illustrations $6 a$ et $6 \mathrm{~b}$ ). La première étape de la démarche a consisté à observer le MNT du bois des Caures et à localiser dans un SIG dix-sept structures de géométrie variée (figure 7). Puis, les coordonnées géographiques de ces dix-sept structures ont été 
transférées vers un DGPS (Trimble GeoXT 3000) équipé d'un écran tactile pour la visualisation de documents numériques. Sur le terrain, la densité du sous-bois a engendré quelques difficultés en termes d'accessibilité, de visibilité et d'exploitation du DGPS, au moment de notre visite sur le terrain. Le recours aux cartes de terrain et à la boussole a été systématique.

Illustrations $6 a$ et $6 \mathrm{~b}$ - Exemple d'une prospection archéologique menée dans la forêt domaniale de Verdun (bois d'Ormont)
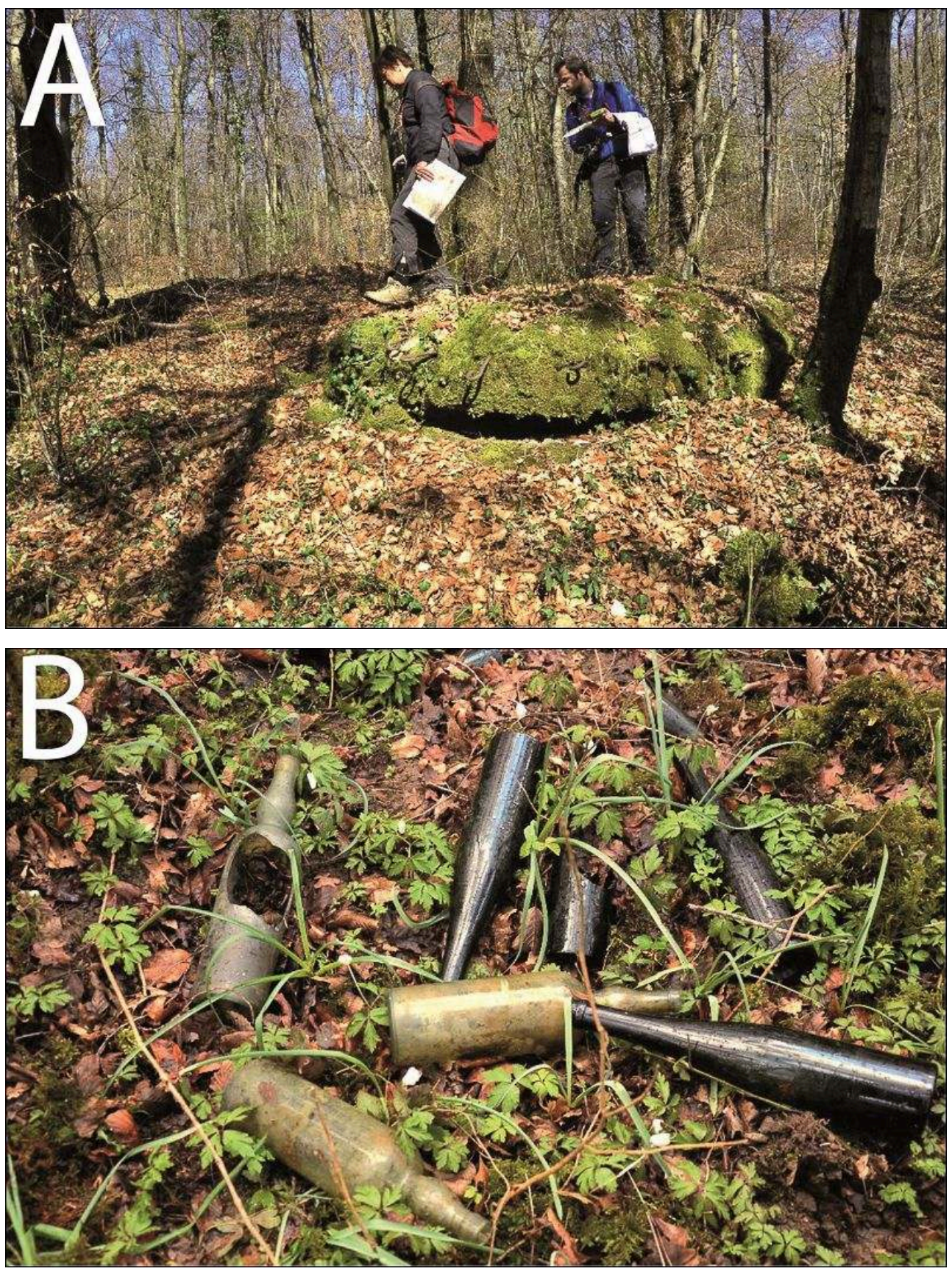

A : Photographie d'un poste d'observation allemand découvert en mars 2014 au sud-ouest du bois d'Ormont, autre secteur de la forêt domaniale de Verdun ayant bénéficié d'une étude archéologique (cliché D. Jacquemot, mars 2014) ; B : Photographie d'une fosse-dépotoir identifiée dans le même secteur, à proximité d'un abri.

Auteur: D. Jacquemot, mars 2014. 
Un premier travail d'observation in situ a consisté à repérer les dix-sept structures relevées sur le MNT pour différencier celles construites - les vestiges de guerre, de géométrie orthogonale, avec présence ou absence de matériaux de construction - des autres polémoformes, notamment les trous d'obus, circulaires ou ellipsoïdaux. Afin d'associer une fonction militaire aux vestiges de guerre, le mobilier archéologique situé à l'intérieur ou à proximité de ces reliefs a été exploité : bouteilles (illustration $6 \mathrm{~b}$ ), matériaux de construction, arbre relique portant traces ou restes de barbelés ou de câbles (Jacquemot et Legendre, 2011). À l'issue de la mission de terrain, chaque structure repérée et interprétée a été manuellement cartographiée dans le SIG.

15 Au bilan, le travail de terrain fut un outil autant de validation que d'interprétation des vestiges identifiés par télédétection. Bien que le couplage archéologie/étude morphologique se soit montré efficace pour répondre aux besoins de l'étude, il fut parfois impossible d'interpréter une structure en raison de sa complexité géométrique (coalescence de plusieurs structures) et/ou de l'absence d'artéfacts. Un moyen de résoudre ce problème a été d'opter pour une vue d'ensemble du secteur d'étude à l'aide du SIG, afin de mieux apprécier les organisations «complexes ». Ainsi, la méthode développée par D. Passmore (2013a) permet d'interpréter ces structures restantes en étudiant leur proximité par rapport à d'autres structures.

\section{Le recours aux archives de la Grande Guerre pour dater les vestiges}

Le travail en archives a été une étape indispensable pour valider le travail d'interprétation et reconstituer la chronologie de la bataille. Les plans directeurs (une vingtaine ont été consultés) au 1/5 000,1/10 000 et 1/20 000, élaborés par le groupe de canevas de tir du Service géographique de l'Armée localisent avec précision la plupart des édifices construits ou projetés par les deux camps (GQGA, 1917 ; Arnould et Simon, 1994 ; Amat, 2001 ; Bèzes, 2014). Une dizaine de prises de vue aériennes de 1916, 1917 et 1918 offre des images synchrones du terrain. Enfin, les cadastres napoléoniens identifient les structures antérieures à la guerre (axes de communication, fossés forestiers). Ces documents, acquis auprès de centres d'archives publics français (Bar-leDuc, Ivry-sur-Seine, Saint-Mandé, Verdun, Vincennes) et un centre d'archives allemand (Dresde), puis dans des fonds privés, conservés par des collectionneurs, sont un élément clé pour l'interprétation du champ de bataille (GQGA, 1917 ; Stichelbaut, 2009, 2011 ; Gheyle et al., 2013 ; Stichelbaut et Chielens, 2013 ; Bèzes, 2014). Afin d'assurer le calage avec le MNT, ces documents ont été géoréférencés puis projetés dans le logiciel ArcGIS (système géodésique RGF93). Leur croisement avec le MNT a affiné le travail d'interprétation, en identifiant uniquement les vestiges de guerre conservés en surface, et d'étudier leur temporalité en collaboration étroite avec les archéologues.

\section{Résultats}

\section{Typologie des vestiges de guerre}

17 La mission de terrain et la visualisation du MNT en pente et à partir de la méthode du Sky-View Factor ont conduit à une première typologie des vestiges observés dans le bois des Caures (illustration 8), qui prenait en compte les caractères morphologiques des 
dix-sept structures échantillonnées (illustration 7), leur mode de construction (terre, pierre, béton) et la présence de mobilier archéologique (Jacquemot et Legendre, 2011).

Illustration 7 - Visualisation LiDAR des dix-sept structures échantillonnées

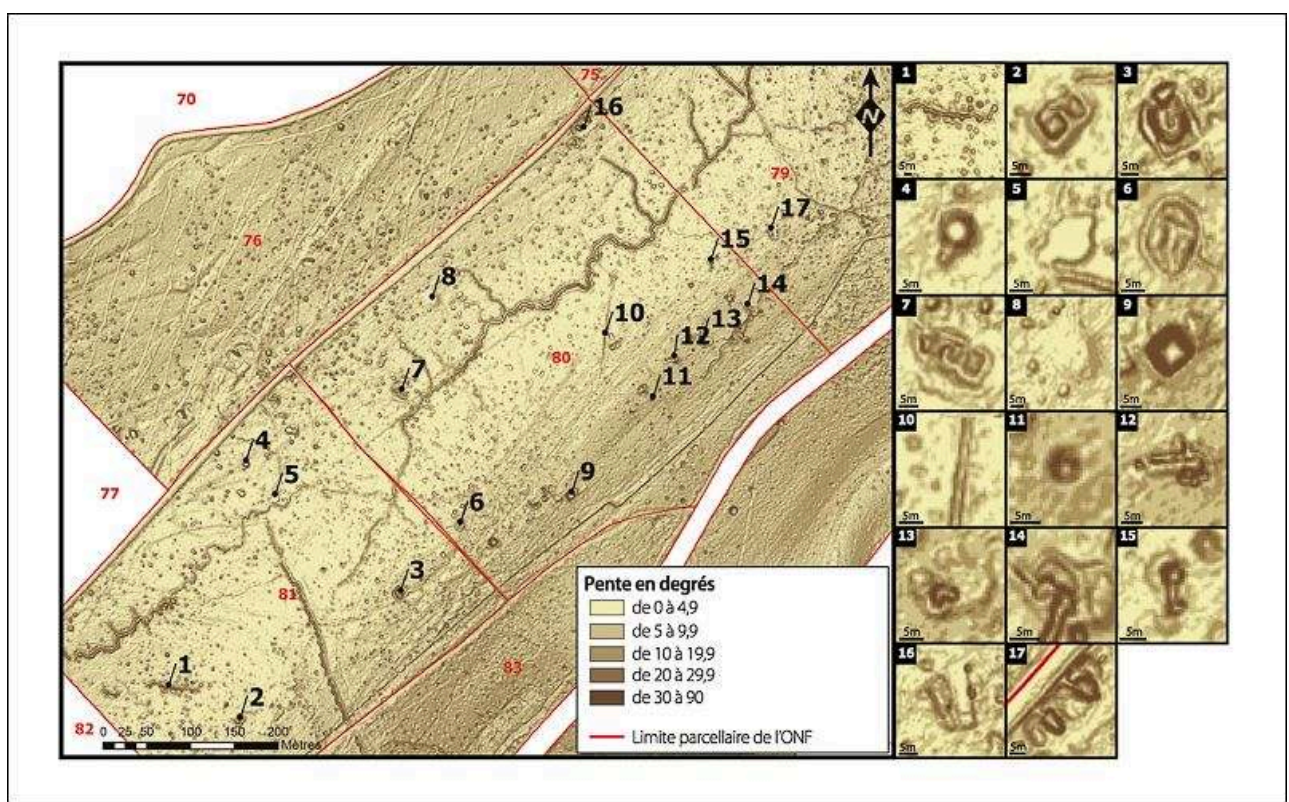

Cette figure localise les dix-sept structures échantillonnées dans le bois des Caures. Les images LiDAR en pente et en Sky-View Factor permettent d'apprécier leur taille et leur morphologie.

Illustration 8 - Typologie dérivée du relevé LiDAR des principaux types de vestiges de guerre visibles dans le bois des Caures

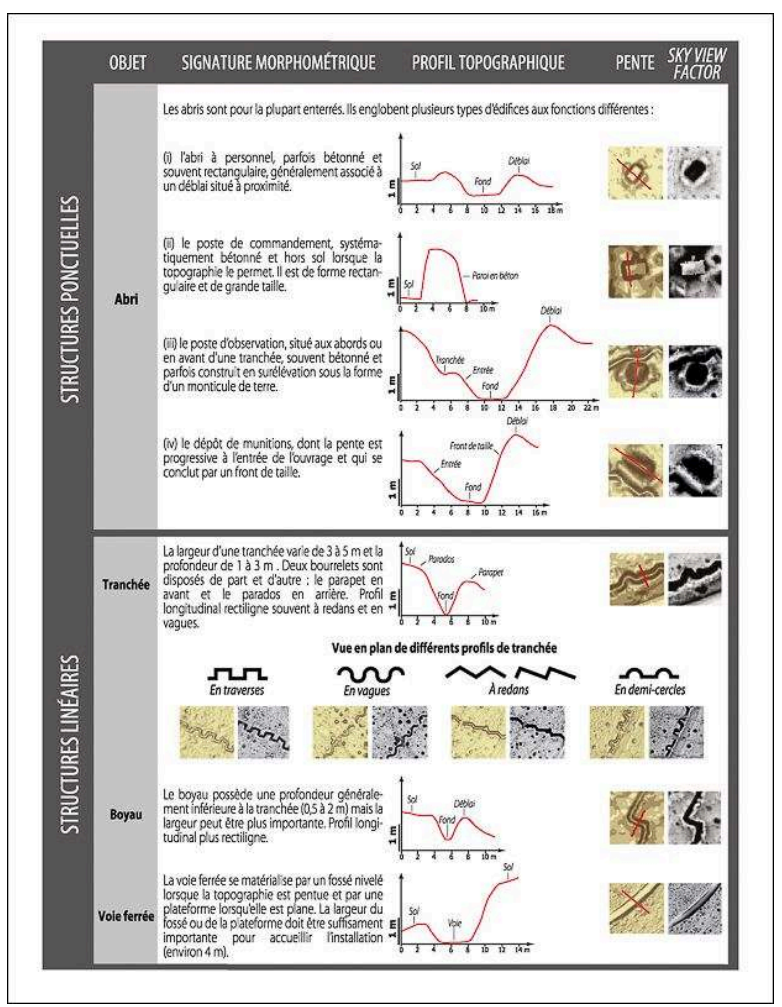

SVF $=$ Sky-View Factor 
Cette typologie dégage deux catégories de vestiges (illustration 8): 1) les vestiges ponctuels associés aux abris de tout type ; 2) les vestiges linéaires assurant les échanges entre l'avant et l'arrière du front et entre les abris.

Chaque catégorie se décline en différentes géométries. Plusieurs facteurs expliquent ces variations morphologiques, qui dépendent surtout de la fonction militaire passée :

-Quatre types de vestiges ponctuels ont été distingués : 1) les abris à personnel, semienterrés, de forme émoussée et polygonale, aux dimensions variées, généralement accompagnés d'un déblai ou d'un bourrelet associé ; 2) les postes de commandement (PC), en surface, semi-enterrés ou souterrains, généralement bétonnés, à la géométrie orthogonale ; 3) les postes d'observation, morphologiquement similaires aux abris à personnel, souvent en avant de la tranchée de première ligne, au plus près de l'ennemi ; 4) les dépôts de munitions, semi-enterrés, dont les entrées excavées se terminent par un front de taille.

- Trois types de vestiges linéaires ont été identifiés : 1) les tranchées, fossés parallèles au front, à redans ou en vagues, d'une largeur de 3 à $5 \mathrm{~m}$, profonds de 1 à $3 \mathrm{~m}$, bordés de deux bourrelets : parapet tourné vers l'ennemi, parados sur l'arrière ; 2) les boyaux, fossés qui connectaient les tranchées entre elles et les organes de la défense, obliques ou perpendiculaires à la ligne de front, souvent moins profonds que les tranchées, parfois plus larges et aux tracés plus rectilignes afin d'accélérer le débit des échanges entre l'avant et l'arrière (Barré, 1917; Solard, 1935; Doyle et Bennett, 1997; Amat, 2001 ; Brantz, 2009 ; Stichelbaut, 2009; Ross, 2011) ; 3) les plates-formes de voie ferrée, peu sinueuses et relativement larges (environ $4 \mathrm{~m}$ ), adaptées à la topographie - fossé ou plate-forme nivelés selon la pente.

D'autres facteurs induisent des variations de géométrie entre des vestiges aux mêmes fonctions. Ainsi intervient le contraste lithologique du plateau des Hauts de Meuse dans la diversité morphologique de certains vestiges. Il a notamment été montré que les modelés étaient moins prononcés dans les placages argileux et marneux de l'Oxfordien supérieur en raison de la proximité de la nappe phréatique, qui rendait difficile le creusement pendant le conflit (Hupy, 2005). L'érosion hydrique post-guerre doit aussi être prise en compte: la stagnation de l'eau facilite l'atterrissement du fond des vestiges et une aggradation de leurs flancs.

\section{Cartographie détaillée du champ de bataille du bois des Caures}

Un travail de généralisation sur les 70 hectares étudiés exploite les critères de reconnaissance et les paramètres morphologiques de la typologie. Il conduit à une carte d'interprétation du champ de bataille du bois des Caures (illustration 9) qui affine la connaissance de la forêt domaniale de Verdun. Cette production cartographique a permis de spatialiser l'ensemble des vestiges de guerre conservés en surface au moment de la mission de télédétection LiDAR (2013). 


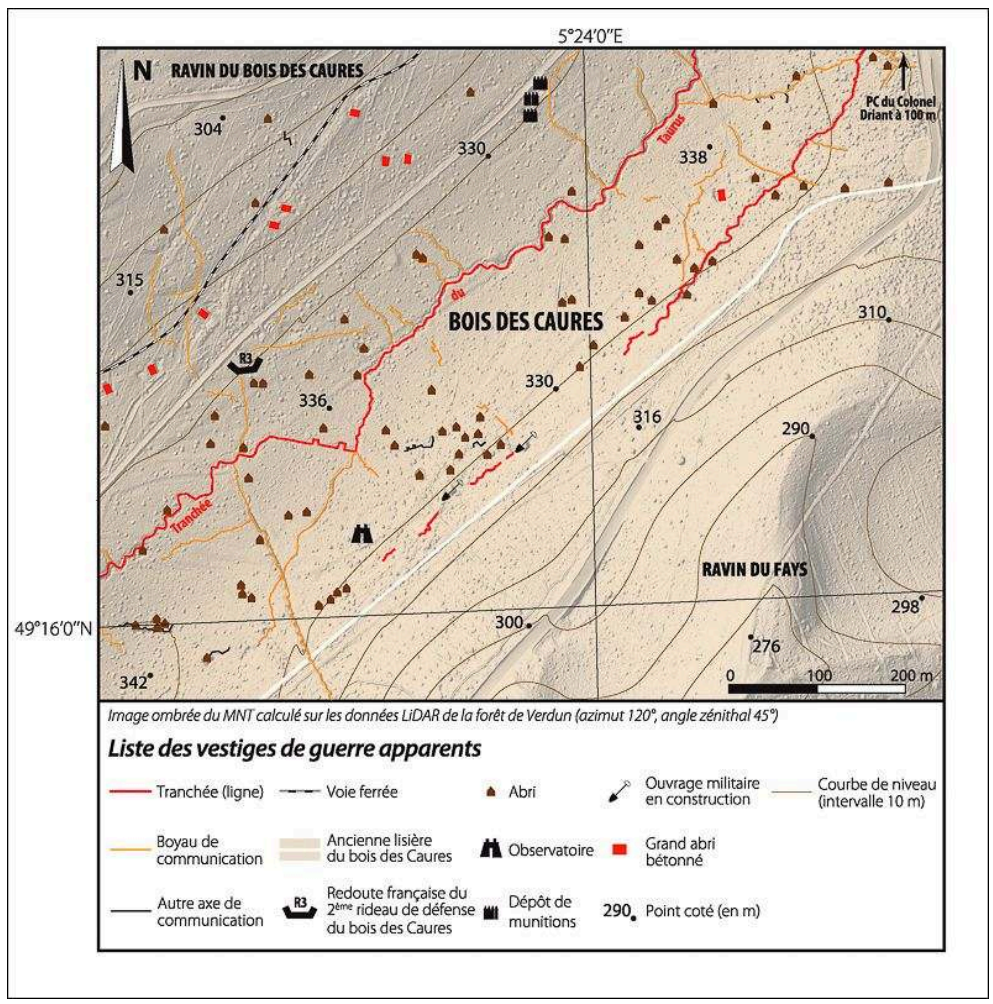

Seules les structures visibles à l'heure actuelle ont été cartographiées.

$\mathrm{Au}$ total, 78 vestiges ponctuels ont été identifiés, comprenant une dizaine de postes d'observation et 9 abris bétonnés. Pour les vestiges linéaires, près de $5 \mathrm{~km}$ de réseaux ont été détectés dont $1,6 \mathrm{~km}$ de tranchées, $2,8 \mathrm{~km}$ de boyaux de communication et $500 \mathrm{~m}$ de voies ferrées.

Au-delà de l'apport du laser aéroporté à l'archéologie, la carte d'interprétation illustre l'organisation du champ de bataille. Le site est un archétype de la Grande Guerre, avec un dispositif de tranchées organisées en lignes et positions et des abris reliés et connectés par ces axes. Du sud-ouest vers le nord-est, le réseau de communications est relativement dense. Une tranchée de première ligne, partiellement achevée et disposée à $100 \mathrm{~m}$ en avant d'une tranchée de deuxième ligne (tranchée du Taurus) a été identifiée; ces deux tranchées coïncident assez bien avec la courbe de niveau $330 \mathrm{~m}$, au-dessus du niveau de débordement de la nappe phréatique. Près d'une dizaine de boyaux grossièrement orthogonaux relient les deux tranchées. Une voie ferrée dans le ravin du bois des Caures alimentait ce secteur du front en matériel et personnel.

Les découvertes confortent les plans directeurs dans l'identification du système défensif allemand : utilisation du béton coffré et des parpaings moulés, techniques bien maîtrisées, bouteilles d'Outre-Rhin, ouvrages gravés en langue allemande (Gaber, 1998 ; Jacquemot et Legendre, 2011 ; Schnitzler et al., 2013). Notons aussi que l'adossement de la tranchée de première ligne à la lisière du bois en durcissait probablement la fonction d'obstacle.

Dans le ravin du Fays, au sud-est du secteur d'étude, l'ancien no man's land est quasiment exempt de vestiges visibles. Ce constat illustre le rôle des bois et des forêts dans la dissimulation des éléments de la défense. Les Allemands ont tiré profit des 
couverts pour y cacher les troupes, les matériels et installer de véritables places fortes, garantissant le maintien des positions en situation de faiblesse. Sur et à proximité de la ligne de contact, le site est criblé de trous d'obus (Barré, 1917 ; Doyle et Bennett, 1997 ; Amat, 2001, 2015; Hupy, 2005; Ross, 2011). Une dizaine de postes de tir et abris d'observation bâtis en arrière de la lisière, à proximité de la tranchée de première ligne, permettaient d'observer en toute discrétion les lignes françaises à quelques centaines de mètres au sud-est du bois. Les abris à personnel s'organisaient en cantonnements en retrait des lignes mais toujours à proximité d'un axe de communication (boyau, voie ferrée). C'est aussi le cas de plus grands édifices, tels les postes de commandement (PC) et les dépôts de munitions (Barré, 1917; Solard, 1935; Stichelbaut, 2009).

\section{Analyse diachronique du champ de bataille dans le bois des Caures}

En complément du travail d'analyse réalisé à partir de la cartographie interprétative du champ de bataille, une analyse diachronique a donné une profondeur temporelle aux résultats acquis. Le croisement des données archéologiques, des images LiDAR, des documents d'archives et du terrain s'est révélé pertinent pour appréhender l'histoire $\mathrm{du}$ bois des Caures pendant les quatre années de guerre (illustration 10). Alors qu'on pouvait poser l'hypothèse de l'existence de vestiges des positions françaises d'avant la bataille, on n'y observe finalement que quelques lignes de retranchement creusées, de l'automne 1915 au début de l'année 1916, par les bataillons de chasseurs à pied: un poste de commandement (PC Driant), dont on aperçoit les abords au nord-est de la carte (illustration 9), puis la redoute R3 à l'ouest, connectée à un boyau de communication et composant le deuxième rideau de défense du bois. 
Illustration 10 - Évolution de l'occupation du bois des Caures entre 1916 et 1918.

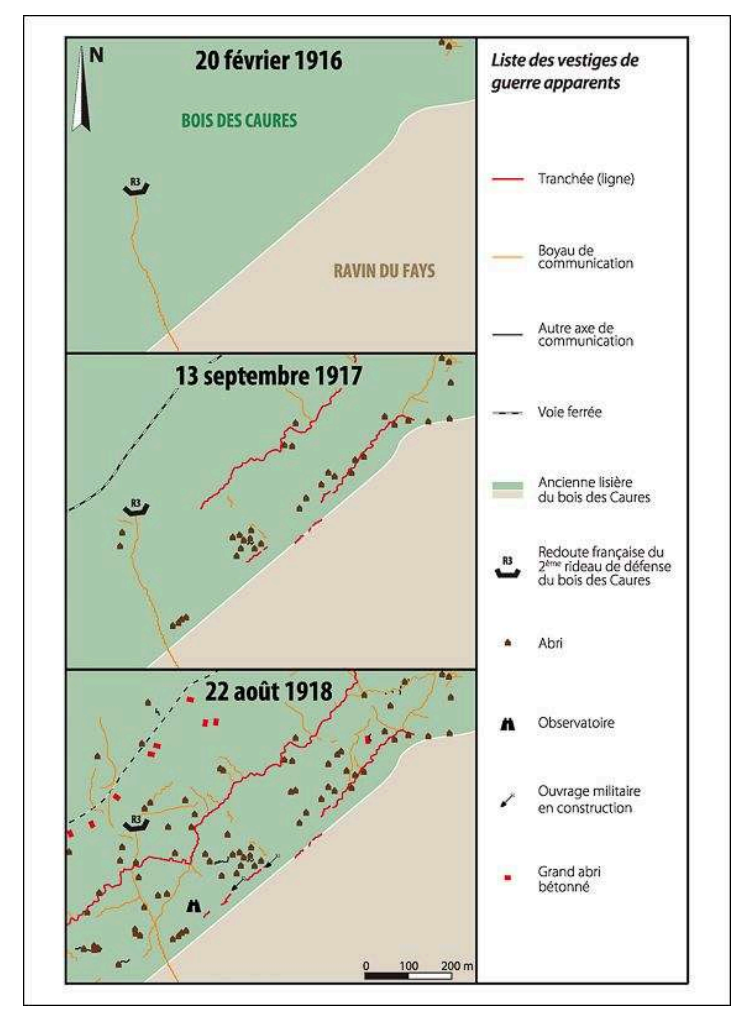

Les pas de temps choisis pour réaliser cette carte se basent sur les dates de tirage d'un plan directeur (groupe des canevas de tir, bois des Caures). Une dizaine de photographies aériennes acquises à des époques intermédiaires ont également été consultées et superposées au LiDAR pour valider les informations du plan directeur.

Sans prétendre aborder les questions stratégiques et opératives, hors du champ de l'étude, ces carences dans le dispositif français pourraient être mises au compte d'un relâchement dans l'effort de fortification de la première position, consécutif au rejet de l'hypothèse d'une offensive allemande sur la place forte de Verdun (Bernède, 2002). À cela s'ajoute le manque d'effectifs disponibles, contraignant l'état-major à employer les réserves, dans un contexte 1) où les troupes usées ne sont plus relevées (Bernède, 2002) et 2) de conditions météorologiques difficiles, faisant de 1916 la sixième année la plus pluvieuse depuis 1877 dans le département de la Meuse (Savouret et al., 2011).

Après la bataille de 1916, les Allemands construisent deux lignes de défense en retrait de la lisière du bois. Le béton, systématiquement utilisé, durcit les positions et les abris se multiplient pendant toute l'année 1917 (Jacquemot et Legendre, 2011 ; Schnitzler et al., 2013). Ils profitent également du ravin du bois des Caures, situé en dehors du champ de vision de l'ennemi, pour y aménager une voie ferrée et des dépôts de munitions. Le réseau des fossés se densifie et les abris se multiplient jusqu'en octobre 1918, dont huit abris bétonnés, distants d'une cinquantaine de mètres environ, le long du versant sud du ravin, sur l'isohypse des $320 \mathrm{~m}$. Ce sont les années « allemandes », 1917 et 1918, qui transformèrent le massif en une véritable forteresse. 


\section{Synthèse et discussion}

29 L'imagerie LiDAR, expérimentée dans le bois des Caures, a démontré sa capacité d'inventaire des vestiges de guerre en contexte infra-forestier. Jusqu'en 2013, le couvert forestier empêchait de cartographier l'ensemble des édifices militaires aménagés sur le champ de bataille. En traversant la canopée, le laser aéroporté a ainsi permis d'identifier des vestiges inconnus à ce jour. 70 nouveaux abris (toutes catégories confondues) ont été détectés par télédétection alors que l'ONF n'en avait à ce jour répertorié que 8 par prospection pédestre (les 8 abris bétonnés du ravin); 4 nouveaux kilomètres de tranchées et de boyaux complètent le réseau d'un kilomètre cartographié par l'ONF (tranchée du Taurus). Les images LiDAR ont aussi révélé une plate-forme de voie ferrée au nord-ouest du secteur d'étude, non renseignée dans la base de données de l'ONF. Enfin, l'étude met en évidence la présence de linéaments propres à l'exploitation forestière post-guerre (cloisonnements), qui peuvent se superposer aux vestiges de tranchées ou de boyaux. Quelques structures n'ont pu être interprétées.

Seule l'approche croisée, archéologique et géomorphologique, a permis l'identification de restes de constructions militaires. Sans observations in situ, ce travail d'interprétation aurait été difficilement réalisable car les images LiDAR ne permettent pas de détecter la présence de mobiliers archéologiques (faible dimension). L'exploitation des plans directeurs et des photographies aériennes a permis l'interprétation de la plupart des vestiges détectés par télédétection. Ces archives pourraient aussi être exploitées pour localiser des structures enfouies ou évaluer le nombre de vestiges conservés en surface, en comparant les infrastructures apparentes sur ces documents et celles relevées sur le MNT. Néanmoins, la fiabilité de ces ressources peut être discutée : informations différentes sur les plans directeurs selon les camps (édifices oubliés lors de la cartographie, édifices maintenus secrets, difficulté à distinguer les édifices construits ou projetés), résolution des photographies aériennes (images floutées ou bruitées par les nuages et aérosols) et manque de visibilité (en particulier les photographies aériennes qui rendent impossible la lecture du champ de bataille sous forêt).

31 Bien que les polémoformes soient facilement identifiables sur le MNT, il reste difficile d'en cartographier l'ensemble. C'est pourquoi, l'étude a focalisé sur les vestiges de guerre, en mettant notamment de côté les reliefs induits par les bombardements. Pour aborder cette question, un travail d'automatisation est en cours, qui permettra de se défaire de la phase de digitalisation, particulièrement lourde. Cette opération permettra la cartographie automatique de tous les reliefs contenus sur le MNT en un temps très court. En diminuant les délais de traitement, la cartographie devrait être généralisée aux 10000 hectares de la forêt domaniale, soit l'ensemble du territoire couvert par la mission LiDAR.

Les cartes d'interprétation et les cartes diachroniques obtenues constituent déjà un apport en matière de connaissance de la forêt domaniale de Verdun, qui n'a été que partiellement cartographiée depuis la reconversion du champ de bataille en territoire forestier au début des années 1930 (Amat, 1987, 2001, 2015). L'étude contribue directement à l'inventaire du patrimoine archéologique, masqué par des décennies de dynamique forestière. Ces ressources iconographiques ainsi que les observations effectuées sur le terrain apportent aussi de nouveaux éléments de réponse quant à l'organisation du dispositif de défense aménagé dans le bois des Caures, en complément 
des précieuses informations contenues sur les plans directeurs et les photographies aériennes.

Dans la perspective de valorisation du site historique et mémoriel de Verdun, l'inventaire et la cartographie présentés dans cet article seront pris en compte dans la prochaine révision (2017-2020) du plan de gestion durable (i.e., plan d'aménagement forestier), permettant d'envisager plus concrètement les modes de gestion conservatoire à mettre en place dans les parcelles. Cela pourrait conduire à 1) préciser et choisir les paramètres techniques de l'exploitation forestière les mieux adaptés aux enjeux identifiés, 2) assurer une meilleure protection et conservation des vestiges de guerre et des polémoformes et 3) valoriser les habitats et écosystèmes spécifiques à une forêt de guerre. Des démarches de ce type ont été déjà été entreprises dans d'autres secteurs du front, notamment en Belgique, où certains sites de mémoire sont inscrits dans une gestion patrimoniale durable (Gheyle et al., 2013).

\section{Conclusions}

Cette étude a permis d'évaluer les potentialités de l'outil LiDAR pour cartographier les vestiges de la Grande Guerre en contexte intra-forestier. Menée sur un champ de bataille à haute valeur historique et archéologique, elle a conduit à une recherche interdisciplinaire mêlant des outils (télédétection, morphométrie) et des champs de la géographie (géomorphologie), de l'archéologie (archéologie de la Grande Guerre) et de l'histoire (Première Guerre mondiale, archives historiques civiles et militaires).

Cette approche croisée a donné lieu à une étude morphologique des vestiges de guerre qui permet d'établir une typologie inédite des vestiges observés dans le bois des Caures. Le résultat de cet examen a conduit à une carte interprétative des 70 hectares de ce bois, apportant de précieux éléments de réponse sur l'état actuel des vestiges de guerre et sur l'organisation spatiale du champ de bataille. La découverte de près de 70 abris et d'environ $4 \mathrm{~km}$ de réseaux de défense enrichit l'inventaire archéologique. Les documents d'archives ont permis de donner, par des cartes diachroniques, des éléments de réponses sur l'évolution temporelle du champ de bataille du bois des Caures.

Cette étude affine les connaissances de la composante Grande Guerre dans les paysages de la forêt domaniale de Verdun. Elle produit dans le même temps des ressources iconographiques directement exploitables en termes de gestion, de conservation et de valorisation patrimoniale. Ce fonds documentaire permettra aux aménageurs de prendre en considération les vestiges de guerre conservés en surface dans le prochain plan d'aménagement forestier, à des fins de conservation et de valorisation.

D'autres secteurs de la forêt sont en cours de prospection. Les résultats acquis sur ces sites alimenteront une base de données pour une typologie des vestiges de guerre, qui devrait aboutir à une cartographie générale des vestiges détectés en forêt domaniale de Verdun. Conduit pour l'instant à partir de quelques sites d'étude, le travail d'analyse spatialisé devrait être étendu et automatisé pour permettre la cartographie des 10000 hectares du massif forestier. Des comparaisons sont envisagées avec d'autres sites de bataille du Front de l'Ouest, afin de mieux apprécier la variété des vestiges de la Grande Guerre. Toutefois, le développement de la méthode présentée dans cet article 
peut s'envisager sur d'autres secteurs d'étude, à partir du moment où l'on a accès à un MNT de haute précision.

\section{BIBLIOGRAPHY}

Amat J.-P., 1987. Guerre et milieux naturels : les forêts meurtries de l'Est de la France, 70 ans après Verdun. L'Espace géographique, 16, 3, p. 217-233.

Amat J.-P., 2001. La forêt entre guerre et paix, 1870-1995 : étude de biogéographie historique sur l'arc meusien de l'Argonne à la Woëvre. Thèse d'État, Lille, Université de Lille 1, 1285 p.

Amat J.-P., 2015. Les forêts de la Grande Guerre : histoire, mémoire, patrimoine. Paris, Presses de l'Université Paris-Sorbonne, 548 p.

Amat J.-P., Foucault Br. de, 1999. Contribution à une monographie historique, floristique et phytosociologique du plateau de Douaumont (forêt domaniale de Verdun). Office national des forêts, Bar-le-Duc, service départemental de la Meuse, $128 \mathrm{p}$.

Arnaud-Fassetta G., Carcaud N. (Eds.), 2015. La géoarchéologie française au XXI ${ }^{\text {èe }}$ siècle. Paris, CNRS Editions, $620 \mathrm{p}$.

Arnould P., 2006. Forêts : deux siècles de crises de la fin du « Petit Âge glaciaire » au début du « Petit Âge chaudière ». In Beck C., Luginbühl Y., Muxart T. (Eds.), Temps et espaces des crises de l'environnement. Versailles, Éditions Quæ, p. 263-276.

Arnould P., Simon L., 1994. Forêts, guerre, après-guerre, autour du Chemin-des-Dames. In Corvol A., Amat J.-P. (Eds.), Forêt et guerre. Paris, Éditions L'Harmattan, p. 251-270.

Barré $C^{\mathrm{dt}}$, 1917. Organisation du terrain. Généralités et organisations défensives. Tome 1, École d'instruction du génie, $47 \mathrm{p}$.

Bernède A., 2002. Verdun 1916 : le point de vue français. Le Mans, Éditions Cénomane, 382 p.

Bèzes B. (Ed.), 2014. 14-18, ou la géographie des désastres. IGN Magazine, n 74, p. 6-20.

Bilodeau C., Deroin J.-P., Sittler B., 2008. Le LiDAR. Apports en archéologie. Archéopages, n² 23, p. 64-77.

Brantz D., 2009. Environments of death: trench warfare on the Western Front, 1914-18. In Closmann C. (Ed.), War and the environment: military destruction in the Modern Age. College Station (Etats-Unis), Texas A\&M University Press, p. 68-91.

Carpentier C., 2004. Géométries et environnements de dépôt de l'Oxfordien de l'Est du Bassin de Paris. Thèse, Nancy, Université Henri Poincaré (Nancy 1), $470 \mathrm{p}$.

Clout H., 1993. The revival of rural Lorraine after the Great War. Geografiska Annaler, 75, 2 , p. 73-91.

Devos A., Taborelli P., Dodici M., Chalumeau L., Buridant J., Bollot N., Combaud A., Desfossés Y., 2015. Rôle des conditions géographiques sur l'organisation spatiale des réseaux de défense de la Grande Guerre. Application à la Champagne (région de Reims). Physio-Géo, n 9, p. 157-174. 
Desfossés Y., Jacques A., Prilaux G. (Eds.), 2008. L'archéologie de la Grande Guerre. Rennes, Éditions Ouest-France/INRAP, $127 \mathrm{p}$.

Doneus M., 2013. Openness as visualization technique for interpretative mapping of airborne LiDAR derived Digital Terrain Models. Remote sensing, 12, 5, p. 6427-6442.

Doneus M., Briese C., 2011. Airborne laser scanning in forested areas - Potential and limitations of an archaeological prospection technique. In Cowley D. (Ed.), Remote sensing for archaeological heritage management. Proceedings of the 11th EAC heritage management symposium. Bruxelles (Belgique), Europae Archaeologia Consilium, p. 53-76.

Doyle P., Bennett M., 1997. Military geography: terrain evaluation and the British Western Front 1914-1918. The Geographical Journal, 163, 1, p. 1-25.

Enay R., Boullier A., 1981. L'âge du complexe récifal des Côtes de Meuse entre Verdun et Commercy et la stratigraphie de l'Oxfordien dans l'Est du Bassin de Paris. Geobios, nº 14, p. 727-771.

Gaber S., 1998. Mémoire de la Grande Guerre en Lorraine. Metz, Éditions Serpenoise, 143 p.

Georges-Leroy M., Bock J., Dambrine E., Dupouey J.-L., 2011. Apport du LiDAR à la connaissance de l'histoire de l'occupation du sol en forêt de Haye. ArchéoSciences, n 35, p. 117-129.

Gheyle W., Dossche R., Bourgeois J., Stichelbaut B., Van Eetvelde V., 2013. Analysis for the cultural heritage management of a World War I militarized landscape: The German field defences in Antwerp. Landscape research, 39, 5, p. 502-522.

Grand Quartier Général des Armées Alliées, 1917. Instruction sur les plans directeurs et les cartes et plans spéciaux (cette instruction abroge l'instruction sur les plans directeurs du 14 février 1916). Paris, Grand Quartier Général des Armées (GQGA) - État-Major, 36 p.

Hesse R., 2010. LiDAR-derived Local Relief Models - a new tool for archaeological prospection. Archaeological prospection, 17, 2, p. 67-72.

Hesse R., 2014. Geomorphological traces of conflict in high-resolution elevation models. Applied geography, $\mathrm{n}^{\circ} 46, \mathrm{p} .11-20$.

Hupy J., 2005. Assessing landscape disturbance and recovery across a WWI battlefield: Verdun, France. Thèse, East Lansing (Etats-Unis), Michigan State University, 209 p.

Hupy J., Schaetzl R., 2006. Introducing « bombturbation », a singular type of soil disturbance and mixing. Soil science, 171, 11, p. 823-836.

Ilyès Z. (Ed.), 2006. Military activities: warfare and defense. In Szabo J., David L., Loczy D. (Eds.), Anthropogenic geomorphology: a guide to man-made landforms. Dordrecht (Pays-Bas), Springer, p. 217-231.

Jacquemot S., Legendre J.-P. (Eds.), 2011. Vestiges de guerres en Lorraine : le patrimoine des conflits mondiaux. Metz, Éditions Serpenoise, $232 \mathrm{p}$.

Kokalj Ž., Zakšek K., Oštir K., 2011. Application of sky-view factor for the visualization of historic landscape features in lidar-derived relief models. Antiquity, 85, 327, p. 263-273.

Masini N., Coluzzi R., Lasaponara R., 2011. On the airborne lidar contribution in archaeology: from site identification to landscape investigation. In Wang C.-C. (Ed.), Laser scanning theory and applications. Rijeka (Croatie), InTech, p. 263-290.

Masson-Loodts I., 2014. Paysages en batailles. Les séquelles environnementales de la Grande Guerre. Bruxelles (Belgique), Éditions Nevicata, 102 p. 
Meuret J.-C., 2010. Archéologie, paysage et histoire d'une forêt du Néolithique à nos jours : le massif de Domnaiche-en-Lusanger (Loire-Atlantique). Annales de Bretagne et des Pays de l'Ouest, 117, 4, p. 7-85.

Passmore D., Tunwell D., Harrison S., 2013a. Landscapes of logistics: the archaeology and geography of WWII German military supply depots in Central Normandy, north-west France. Journal of conflict archaeology, 8, 3, p. 165-192.

Passmore D., Harrison S., Tunwell D., 2013b. Second World War conflict archaeology in the forests of north-west Europe. Antiquity, 88, 342, p. 1275-1290.

Rey F., 2003. Influence of vegetation distribution on sediment yield in forested marly gullies. Catena, $\mathrm{n}^{\circ} 50$, p. 549-562.

Robert S. (Ed.), 2011. Sources et techniques de l'archéogéographie. Besançon, Presses Universitaires de France-Comté, 246 p.

Ross W., 2011. Landscapes of the Western Front: Materiality during the Great War. New York (EtatsUnis), Routledge, 244 p.

Savouret E., Amat J.-P., Cantat O., Filippucci P., 2011. Au temps météorologique de la Grande Guerre : approche séquentielle des périodes contraignantes dans les tranchées sur le front de la Marne et de la Meuse, 1914-1918. Climatologie, n 8, p. 59-77.

Schnitzler B., Landolt M. (Eds.), 2013. À l'est, du nouveau ! Archéologie de la Grande Guerre en Alsace et en Lorraine. Strasbourg, Éditions des Musées de la Ville de Strasbourg, 368 p.

Solard $C^{\text {ne }}$, 1935. Fortification. La fortification pendant la guerre 1914-1918. Tome 2, Fontainebleau, École d'Application d'Artillerie, 148 p.

Stéphane M., 1929. Ma dernière relève au bois des Caures. Paris, Cabinet du Pamphlétaire, 223 p. Stichelbaut B., 2009. World War One aerial photography: an archaeological perspective. Thèse, Gand (Belgique), Universiteit Gent, 434 p.

Stichelbaut B., 2011. The first thirty kilometers of the western front 1914-1918: an aerial archaeological approach with historical remote sensing data. Archaeological prospection, 18, p. 57-66.

Stichelbaut B., Chielens P., 2013. La guerre vue du ciel : 1914-1918, le front en Belgique. Bruxelles (Belgique), Fonds Mercator, $350 \mathrm{p}$.

Štular B., Kokalj Ž., Oštir K., Nuninger L., 2012. Visualization of lidar-derived models for detection of archaeological features. Journal of archaeological science, 39, 11, p. 3354-3360.

Thornbush M., 2012. Archaeogeomorphology as an application in physical geography. Applied geography, n 34, p. 325-330.

Van Hollebeeke Y., Stichelbaut B., Bourgeois J., 2012. From landscape of war to archaeological report: ten years of professional World War I archaeology in Flanders (Belgium). European Journal of Archaeology, 17, 4, p. 702-719.

Wandsnider L., 1992. Archaeological landscape studies. In Rossignol J., Wandsnider L. (Eds.), Space, time, and archaeological landscapes. New York (Etats-Unis), Springer, p. 285-292.

Zakšek K., Oštir K., Kokalj Ž., 2011. Sky-View Factor as a relief visualization technique. Remote sensing, 12, 3, p. 398-415. 


\section{ABSTRACTS}

A century after the Great War, traces of the fighting remain numerous in the landscapes of North-Eastern France. In 2013, an airborne LiDAR survey conducted in the Verdun forest allowing us to highlight hidden and preserved remnants of war on 3-D images. This study explores the potential of LiDAR in analysing and mapping conserved traces of military facilities in the Caures wood (Verdun forest), site of the first fights of the Verdun battle (1916). Data processing and fieldwork serve to establish an innovative typology and detailed maps of remnants diversity, shelters and ditches, and organisation of the battlefield.

Cent ans après la Grande Guerre, les traces des champs de bataille restent présentes dans les paysages du Nord-Est de la France. En 2013, une prospection par LiDAR aéroporté de la forêt domaniale de Verdun a mis en évidence sous forme d'images 3-D à haute résolution les vestiges de guerre masqués par le manteau forestier. Cette étude explore les potentialités de l'outil LiDAR pour l'analyse et la cartographie des témoins des aménagements militaires dans le bois des Caures (forêt domaniale de Verdun), lieu des premiers combats de la bataille de Verdun (1916). Le traitement d'images combiné à l'analyse de terrain permet d'établir une typologie et une cartographie inédites de la diversité des vestiges, abris et fossés, et de l'organisation du champ de bataille.

\section{INDEX}

Mots-clés: LiDAR aéroporté, vestige de guerre, polémoforme, carte d'interprétation, document d'archives

Keywords: airborne LiDAR, remnant of war, conflict-induced landform, interpretative mapping, archival document

\section{AUTHORS}

\section{RÉMI DE MATOS MACHADO}

Rémi de Matos Machado, remi.machado@univ-paris-diderot.fr, est doctorant contractuel en géographie physique avec mission d'enseignement à l'université Paris-Diderot (Paris 7) et membre de l'UMR 8586 PRODIG (Pôle de Recherches pour l'Organisation et la Diffusion de l'Information Géographique).

\section{JEAN-PAUL AMAT}

Jean-Paul Amat est professeur émérite de géographie de l'université Paris Sorbonne, ancien directeur (2006-2010) de l'UMR 8185 ENEC (Espaces, Nature et Culture).

\section{GILLES ARNAUD-FASSETTA}

Gilles Arnaud-Fassetta est professeur de géographie (hydrogéomorphologie, géoarchéologie) à l'université Paris-Diderot (Paris 7) et membre de l'UMR 8586 PRODIG.

\section{FRANÇOIS BÉTARD}

François Bétard est maître de conférences en géographie physique à l'université Paris-Diderot (Paris 7) et membre de l'UMR 8586 PRODIG. 